\title{
Lyophilized Powder Dosage Form
}

National Cancer Institute

\section{Source}

National Cancer Institute. Lyophilized Powder Dosage Form. NCI Thesaurus. Code C87541.

A pharmaceutical dosage form comprised of lyophilized powder. 